\title{
MICROALBUMINURIA Y RETINOPATÍA DIABÉTICA
}

\section{MICROALBUMINURIA AND DIABETIC RETINOPATHY}

\author{
ASENSIO-SÁNCHEZ VM ${ }^{1}$, RODRÍGUEZ-DELGADO B ${ }^{2}$, GARCÍA-HERRERO E ${ }^{2}$, \\ CABO-VAQUERA V ${ }^{2}$, GARCÍA-LOYGORRI C ${ }^{1}$
}

\section{RESUMEN}

Objetivo: Estudiar la prevalencia de microalbuminuria y su asociación con las formas más severas de retinopatía diabética en una población de diabéticos insulinodependientes.

Material y método: Se estudiaron 360 pacientes con diabetes insulinodependiente con al menos cinco años de evolución en un periodo comprendido entre enero de 1998 y diciembre de 2005. Se evaluó la presencia de microalbuminuria por inmunoanálisis. Los pacientes fueron estudiados con oftalmoscopía directa e indirecta y clasificados como no retinopatía, retinopatía no proliferante, retinopatía no proliferante severa / proliferante y edema macular. Resultados: En este estudio el 24,1\% de los pacientes tenían microalbuminuria. La mayoría de los pacientes con microalbuminuria y macroalbuminuria eran varones con mayor tiempo de evolución de la diabetes. La microalbuminuria se asoció con las formas más severas de retinopatía diabética.

Conclusiones: Todos los pacientes diabéticos insulinodependiente con al menos cinco años de evolución deberían ser evaluados en su función renal incluida la microalbuminuria y los pacientes con microalbuminuria deberían ser revisados más frecuentemente.

Palabras clave: Diabetes insulinodependiente, microalbuminuria, macroalbuminuria, retinopatía diabética, ceguera legal, hipertensión arterial.

\section{ABSTRACT}

Objective: To study the prevalence of microalbuminuria and its association with more severe diabetic retinopathy in a group of insulin-dependent diabetic patients.

Materials and methods: During the period of January 1998 to December 2005 we examined 360 insulin-dependent diabetic patients with at least five years of evolution. We evaluated the presence of microalbuminuria by immunoanalysis. Patients were evaluated by direct and indirect ophthalmoscopy and classified as non-retinopathy, non-proliferative, severe non-proliferative/proliferative, or macular edema.

Results: In this study, $24.1 \%$ of patients had microalbuminuria. Most of the patients with microalbuminuria and macroalbuminuria were male and had a longer history of diabetes. Microalbuminuria was associated with more severe diabetic retinopathy.

Conclusions: All patients with insulin-dependent diabetes of at least five years' evolution should undergo an evaluation of renal function including tests for microalbuminuria. In the presence of microalbuminuria an ophthalmologic follow-up may be particularly important (Arch Soc Esp Oftalmol 2007; 82: 85-88).

Key words: Insulin-dependent diabetes, microalbuminuria, macroalbuminuria, diabetic retinopathy, legal blindness, arterial hypertension.

\footnotetext{
Recibido: 2/11/06. Aceptado: 17/9/07.

Hospital General. Medina del Campo. Valladolid. España.

1 Doctor en Medicina.

2 Licenciado en Medicina.

Correspondencia:

V.M. Asensio Sánchez

Hospital General Servicio Castellano-Leonés de Salud

Servicio de Oftalmología

Medina del Campo (Valladolid)

España

E-mail: vasensio@hmdc.sacyl.es
} 


\section{INTRODUCCIÓN}

Los pacientes con diabetes mellitus insulinodependiente desarrollan nefropatía diabética en el $40 \%$ de los casos y hasta el $50 \%$ una forma severa de retinopatía diabética después de 20 años de evolución, siendo responsable de hasta un $30 \%$ de las cegueras en la población activa de los países industrializados $(1,2)$. La prevalencia de microalbuminuria entre los pacientes diabéticos es del 15-20\%. La persistencia de microalbuminuria en los diabéticos es un marcador de riesgo no sólo para enfermedad renal y cardiovascular sino para morbilidad ocular severa $(2,3)$.

El objeto de este trabajo es establecer la relación entre microalbuminuria y retinopatía en diabéticos adultos insulinodependientes con más de cinco años de evolución y evaluar su influencia en el desarrollo de retinopatía diabética.

\section{SUJETOS, MATERIAL Y MÉTODOS}

Este estudio fue aprobado por la Comisión de Investigación y Docencia de nuestro hospital. Los pacientes se reclutaron de la consulta de retina y de la consulta general. Se estudiaron 360 pacientes diabéticos insulinodependientes entre los años 1998 y 2005. En la tabla I se muestran las características demográficas de la población estudiada. Los criterios de inclusión para los pacientes fueron los siguientes:

- Aceptar participar en el estudio con firma del consentimiento informado.

- Diabetes mellitus insulinodependiente tratada por endocrino, internista o médico de atención primaria, con cinco o más años de evolución.

- Poder acceder a su historial clínico.

- No haber sido tratado tres meses previos al estudio oftalmológico con corticoides, fármacos nefrotóxicos y/o con un proceso quirúrgico.

- No estar en tratamiento nefrológico por insuficiencia renal crónica y/o diálisis.

Tabla I. Características demográficas

\begin{tabular}{l}
\hline N. $\quad 360$ \\
Sexo (H/M) $\quad 190 / 170$ \\
Edad: 59,2 DE 11,3 \\
Edad de inicio de la diabetes: 39 DE 10 años \\
Tiempo de evolución de la diabetes: 19 DE 10 años \\
\hline
\end{tabular}

H: hombres; M: mujeres; DE: desviación estándar.
En todos los pacientes se realizó un estudio de fondo de ojo bajo midriasis por un mismo oftalmólogo y fueron clasificados según el grado de retinopatía en: 1) no retinopatía $(n=90), 2)$ retinopatía no proliferativa $(n=90), 3)$ maculopatía $(n=90)$, 4) retinopatía no proliferante avanzada o retinopatía proliferante $(n=90)$.

A los pacientes se les dieron instrucciones para recoger la orina de 24 horas. La albuminuria fue determinada en el laboratorio por radioinmunoensayo. Los valores de albuminuria en el rango de micro o macroalbuminuria fueron confirmados en un segundo estudio de orina antes de ser considerados.

\section{Definiciones}

Microalbuminuria: excreción urinaria de albúmina entre $30 \mathrm{mg} / 24$ horas y $300 \mathrm{mg} / 24$ horas, independientemente del método de recolección de orina.

Macroalbuminuria: excreción urinaria de albúmina por encima de $300 \mathrm{mg} / 24$ horas, independientemente del método de recolección de orina.

Ceguera legal: cuando la visión máxima corregida en el mejor ojo es menor o igual a 0,1 .

Hipertensión arterial: si el paciente estaba con tratamiento antihipertensivo o con cifras superiores a 140/90 $\mathrm{mmHg}$.

El estudio estadístico se realizó con un programa de análisis (SAS/STAT) aplicando el test de chicuadrado. Se calculó la asociación entre microalbuminuria y retinopatía mediante el cálculo de la odds ratio (OR) y su correspondiente intervalo de confianza a un grado de confianza del 95\%. Con regresión logística, se evaluó el riesgo dependiente de la microalbuminuria, considerando como variable dependiente o respuesta la presencia o ausencia de retinopatía. Los resultados se consideraron significativos para un nivel de confianza del $95 \%$.

\section{RESULTADOS}

En nuestra población de diabéticos insulinodependientes (tabla I) el $24,1 \%$ de los pacientes tenían microalbuminuria, siendo mayor en los pacientes diabéticos con más tiempo de evolución y en los hombres $(\mathrm{OR}=4,2$; IC 95\%=1,88-6,1) que en las mujeres $(\mathrm{OR}=3,6 ; 95 \% \mathrm{IC}=1,42-5,1)$ (tabla II). Los pacientes con albuminuria tenían formas más 
Tabla II. Albuminuria en función del tiempo de evolución

\begin{tabular}{lccc}
\hline & NA & MA & MAA \\
\hline H/M & $80 / 106$ & $48 / 39$ & $50 / 37$ \\
$\begin{array}{l}\text { Tiempo evolución } \\
\text { (DE años) }\end{array}$ & 9,2 (DE 2) & 16,3 (DE 10) & 18,8 (DE 5) \\
\hline \hline
\end{tabular}

H: hombre; M: mujer; NA: no albuminuria; MA: microalbuminuria; MAA: macroalbuminuria; DE: desviación estándar.

severas de retinopatía (maculopatía, no proliferante avanzada o proliferante) y de ceguera por diabetes mellitus $(\mathrm{p}<0,01)$ (tablas III, IV) siendo el 19,72\% del total ciegos legales. De los 90 pacientes con retinopatía severa el $37,78 \%(n=34)$ y el $51,11 \%$ $(n=46)$ presentaban microalbuminuria y macroalbuminuria respectivamente. De los pacientes con edema macular $(n=90)$ el $31,1 \% \quad(n=28)$ y el $40 \%$ $(\mathrm{n}=36)$ tenían microalbuminuria y macroalbuminuria respectivamente. El riesgo de retinopatía proliferante se incrementó con la duración de la diabetes, pero se aceleró en los pacientes con microalbuminuria y especialmente en los que tenían albuminuria $(\mathrm{OR}=4,2 ; 95 \% \mathrm{IC}=1,88-6,1(\mathrm{p}<0,001)) . \mathrm{La}$ probabilidad de padecer retinopatía severa se incrementa en un factor de 2,6 veces frente al paciente que no tiene microalbuminuria (IC 95\%=1,2-3,6; $\mathrm{p}<0,001)$. La hipertensión arterial fue más frecuente en los casos de maculopatía y retinopatía proliferante que en los otros dos grupos $(\mathrm{p}<0,001)$ y se asoció a niveles de presión sistólica más elevados.

\section{DISCUSIÓN}

La ceguera es considerada la más temida de las complicaciones de la enfermedad humana a excepción de la muerte. En 2002, 124 millones de personas tenían baja visión y 37 millones estaban ciegos (4). En los países occidentales la diabetes mellitus es la primera causa de pérdida de visión en los pacientes jóvenes $(4,5)$. La retinopatía diabética es la más grave de las múltiples complicaciones de la diabetes a nivel ocular y aunque los avances en su tratamiento han mejorado en los últimos 40 años, al ser la incidencia de diabetes mellitus entre el 5 y el $6 \%$ de la población, la retinopatía sigue siendo un gran problema médico-social (5). Alrededor de 1 de cada 10 diabéticos es insulinodependiente y después de 20 años de evolución casi el 100\% desarrollará algún grado de retinopatía diabética y hasta el $50 \%$ desarrollará una forma severa de retinopatía (1). La albúmina es una proteína plasmática, la más abundante de ellas, con un peso molecular aproximado de $69.000 \mathrm{D}$ y es la mayor responsable de la presión coloidosmótica total del plasma. El valor normal de excreción urinaria de albúmina es menor a $30 \mathrm{mg} / 24$ horas, considerándose microalbuminuria a la pérdida por orina entre $30-300 \mathrm{mg} / 24$ horas y que no son detectados por los métodos analíticos convencionales que se utilizan en el laboratorio para el estudio de proteínas (6). La presencia de microalbuminuria de forma repetida en las muestras de orina de un diabético indica daño en la membrana basal glomerular y debe considerarse como una nefropatía diabética precoz. En la base de datos Medline existen 364 artículos que hacen referencia a microalbuminuria diabética y retinopatía diabética de los cuales una minoría se refieren a los diabéticos insulinodependientes. En nuestra población

Tabla IV. Prevalencia de ceguera en función del tipo de retinopatía y albuminuria

\begin{tabular}{lcccc}
\hline & NRD & RDNP & MD & RDNPs+RDP \\
\hline NA & 0 & 0 & $2(2,22 \%)$ & $9(10 \%)$ \\
MA & 0 & 0 & $6(6,67 \%)$ & $12(13,33 \%)$ \\
MAA & 0 & 0 & $13(14,44 \%)$ & $29(32,22 \%)$ \\
\hline \hline
\end{tabular}

NRD: No retinopatía diabética; RDNP: Retinopatía diabética no proliferante; MD: Maculopatía diabética; RDNPs: Retinopatía diabética no proliferante severa; RDP: Retinopatía diabética proliferante; NA: No albuminuria; MA: Microalbuminuria; MAA: Macroalbuminuria.

Tabla III. Prevalencia de albuminuria en función del tipo de retinopatía

\begin{tabular}{lcccc}
\hline & NRD & RDNP & MD & RDNPs+RDP \\
90 & 90 & 90 & $10(11,11 \%)$ \\
NA & 90 & $69(76,67 \%)$ & $26(28,89 \%)$ & $34(37,78 \%)$ \\
MA & $74(82,22 \%)$ & $18(20 \%)$ & $28(31,11 \%)$ & $46(51,11 \%)$ \\
MAA & $16(17,78 \%)$ & $3(3,33 \%)$ & $36(40 \%)$ & $50 / 40$ \\
TA (Sí/No) & $0(0 \%)$ & $9 / 81$ & $40 / 50$ & \\
\hline \hline
\end{tabular}

NRD: No retinopatía diabética; RDNP: Retinopatía diabética no proliferante; MD: Maculopatía diabética; RDNPs: Retinopatía diabética no proliferante severa; RDP: Retinopatía diabética proliferante; NA: No albuminuria; MA: Microalbuminuria; MAA: Macroalbuminuria; TA: Tensión arterial. 
diabética la incidencia de microalbuminuria fue del $24,1 \%$, equivalente a la de otros trabajos $(6,7)$. Los pacientes se caracterizaron por ser fundamentalmente varones, con historia médica de diabetes mellitus precoz y de mayor duración. En este estudio el tipo de retinopatía se correlacionó con el grado de albuminuria, los pacientes con microalbuminuria tenían 2,6 veces más posibilidad de tener retinopatía severa que los que no tenían microalbuminuria; sin embargo el $11,11 \%$ de los pacientes con retinopatía grave tenían una excreción normal de albúmina, mientras el $20,56 \%$ de los pacientes con grados menos severos tenían excreción anómala de albúmina, de aquí que no siempre exista una correlación entre la lesión vascular renal y la retiniana, aunque los pacientes con nefropatía diabética normalmente presentan formas más severas de retinopatía diabética $(2,3)$. Hoy se piensa que la microangiopatía renal precede a la retiniana, por lo que los diabéticos con microalbuminuria deben ser controlados especialmente (7). A pesar de la conocida asociación entre estadios avanzados de retinopatía diabética y macroalbuminuria o proteinuria franca, la relación con microalbuminuria es contradictoria. Aroca PR y col. (8) en un estudio prospectivo a 5 años en 104 pacientes diabéticos tipo 1 determinaron que la microalbuminuria no era un buen marcador para la retinopatía diabética aunque en un artículo posterior (9) consideran que su presencia debe indicar estudios de fondo de ojo más repetidos. Cruickshanks KJ et al. (7) establecen que la microalbuminuria puede ser un marcador de riesgo para retinopatía proliferante. La microalbuminuria indicaría un estado de disfunción vascular generalizada, y microalbuminuria y retinopatía diabética tienen determinantes comunes como la duración de la diabetes y los niveles de tensión arterial. La hipertensión arterial fue más frecuente en los casos severos y se asoció a niveles de presión sistólica más elevados. La ventaja de este estudio es que los pacientes fueron incluidos cumpliendo los criterios establecidos en sujetos, material y método, frente a los estudios de hospitales de referencia donde hay una selección de los pacientes a favor de las formas más severas de diabetes y sus complicaciones, por eso nuestro trabajo refleja el estado de una población básica de ámbito rural.

En los pacientes diabéticos insulinodependientes el estudio de la albúmina en orina debe ser rutinario, porque la presencia de microalbuminuria es un factor de riesgo cardiovascular, renal y de mal pronóstico en la retinopatía que se asocia a ceguera y mortalidad $(2,6,7)$; además la progresión de la microalbuminuria se puede detener en sus estadios iniciales con un mejor control de la diabetes, con dieta pobre en proteínas y con inhibidores de la angiotensina, y los diabéticos con microalbuminuria se beneficiarían de un mayor seguimiento oftalmológico.

\section{BIBLIOGRAFÍA}

1. Ferris FL 3rd, Davis MD, Aiello LM. Treatment of diabetic retinopathy. $N$ Engl J Med 1999; 341: 667-678.

2. Deckert T, Kofoed-Enevoldsen A, Norgaard K, BorchJohnsen K, Feldt-Rasmussen B, Jensen T. Microalbuminuria. Implications for micro- and macrovascular disease. Diabetes Care 1992; 15: 1181-1191.

3. Damsgaard EM, Froland A, Jorgensen OD, Mogensen CE. Microalbuminuria as predictor of increased mortality in elderly people. BMJ 1990; 300: 297-300.

4. Aiello LP. Angiogenic pathways in diabetic retinopathy. $N$ Engl J Med 2005; 353: 839-841.

5. Frank RN. Diabetic retinopathy. N Engl J Med 2004; 350: 48-58.

6. Miccoli R, Giampietro O, Penno G, Odello G, Anichini R, Bertolotto A, et al. «Microalbuminuria» in type I (insulindependent) diabetic patients with and without retinopathy. Acta Diabetol Lat 1989; 26: 163-170.

7. Cruickshanks KJ, Ritter LL, Klein R, Moss SE. The association of microalbuminuria with diabetic retinopathy. The Wisconsin Epidemiologic Study of Diabetic Retinopathy. Ophthalmology 1993; 100: 862-867.

8. Aroca PR, Espeso Sentis O, Del Castillo DD. Estudio prospectivo de correlación entre retinopatía diabética y microalbuminuria en pacientes diabéticos tipo 1. Arch Soc Esp Oftalmol 2000; 75: 307-314.

9. Romero Aroca P, Espeso Sentís O, Sardá Aure P, del Castillo Déjardin D. Relación entre microalbuminuria y retinopatía diabética en diabetes mellitus tipo 1. Rev Clin Esp 2000; 200: 351-354. 